\title{
Front Matter: Volume 6658
}

, "Front Matter: Volume 6658," Proc. SPIE 6658, Organic Field-Effect Transistors VI, 665801 (21 September 2007); doi: 10.1117/12.773658

SPIE Event: Photonic Devices + Applications, 2007, San Diego, California, United SPIE. States 


\section{PROCEEDINGS OF SPIE}

\section{Organic Field-Effect Transistors VI}

Zhenan Bao

David J. Gundlach

Editors

26-28 August 2007

San Diego, California, USA

Sponsored and Published by

SPIE

Volume 6658 
The papers included in this volume were part of the technical conference cited on the cover and title page. Papers were selected and subject to review by the editors and conference program committee. Some conference presentations may not be available for publication. The papers published in these proceedings reflect the work and thoughts of the authors and are published herein as submitted. The publisher is not responsible for the validity of the information or for any outcomes resulting from reliance thereon.

Please use the following format to cite material from this book:

Author(s), "Title of Paper," in Organic Field-Effect Transistors VI, edited by Zhenan Bao, David J. Gundlach, Proceedings of SPIE Vol. 6658 (SPIE, Bellingham, WA, 2007) Article CID Number.

ISSN 0277-786X

ISBN 9780819468062

Published by

SPIE

P.O. Box 10, Bellingham, Washington 98227-0010 USA

Telephone +1 3606763290 (Pacific Time) · Fax +1 3606471445

SPIE.org

Copyright (C 2007, Society of Photo-Optical Instrumentation Engineers

Copying of material in this book for internal or personal use, or for the internal or personal use of specific clients, beyond the fair use provisions granted by the U.S. Copyright Law is authorized by SPIE subject to payment of copying fees. The Transactional Reporting Service base fee for this volume is $\$ 18.00$ per article (or portion thereof), which should be paid directly to the Copyright Clearance Center (CCC), 222 Rosewood Drive, Danvers, MA 01923. Payment may also be made electronically through CCC Online at copyright.com. Other copying for republication, resale, advertising or promotion, or any form of systematic or multiple reproduction of any material in this book is prohibited except with permission in writing from the publisher. The CCC fee code is $0277-786 \times / 07 / \$ 18.00$.

Printed in the United States of America.

Publication of record for individual papers is online in the SPIE Digital Library.

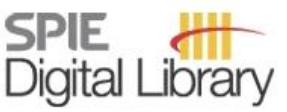

SPIEDigitallibrary.org

Paper Numbering: Proceedings of SPIE follow an e-First publication model, with papers published first online and then in print and on CD-ROM. Papers are published as they are submitted and meet publication criteria. A unique, consistent, permanent citation identifier (CID) number is assigned to each article at the time of the first publication. Utilization of CIDs allows articles to be fully citable as soon they are published online, and connects the same identifier to all online, print, and electronic versions of the publication. SPIE uses a six-digit CID article numbering system in which:

- The first four digits correspond to the SPIE volume number.

- The last two digits indicate publication order within the volume using a Base 36 numbering system employing both numerals and letters. These two-number sets start with 00, 01, 02, 03, 04, 05, $06,07,08,09,0 A, 0 B \ldots 0 Z$, followed by $10-12,20-2 Z$, etc.

The CID number appears on each page of the manuscript. The complete citation is used on the first page, and an abbreviated version on subsequent pages. Numbers in the index correspond to the last two digits of the six-digit CID number. 


\section{Contents}

vii Conference Committee

\section{SESSION 1 CIRCUITS AND DISPLAYS}

665802 Capacitance-voltage characteristics of organic thin-film transistors (Invited Paper) [6658-01]

G. H. Gelinck, TNO (Netherlands); E. van Veenendaal, Polymer Vision (Netherlands);

H. van der Vegte, R. Coehoorn, Philips Research (Netherlands)

665803 Organic thin-film transistor arrays for active-matrix organic light emitting diode (Invited Paper) [6658-02]

S. Lee, H. Moon, D. H. Kim, B.-W. Koo, E.-J. Jeong, B.-L. Lee, J.-Y. Kim, E. Lee, K.-M. Hahn, J.-S. Han, J.-I. Park, J.-B. Seon, J.-W. Kim, Y.-T. Chun, S. Kim, S. K. Kang, Samsung Advanced Institute of Technology (South Korea)

$665804 \quad 13.56 \mathrm{MHz}$ polymer rectifier by printing processes (Invited Paper) [6658-03]

C.-Y. Lin, C.-H. Chou, J. Hou, Y.-J. Chan, Industrial Technology Research Institute (Taiwan)

\section{SESSION 2 GATE DIELECTRICS AND CONTACTS}

665807 High performance organic field-effect transistors with fluoropolymer gate dielectric [6658-06]

W. L. Kalb, T. Mathis, S. Haas, A. F. Stassen, B. Batlogg, ETH Zürich (Switzerland)

\section{SESSION 3 CHARGE TRANSPORT}

6658 0C Determination of the density of trap states in organic thin film transistors [6658-1 1]

F. Yan, The Hong Kong Polytechnic Univ. (Hong Kong China)

6658 OD Charge transport mechanisms in organic and microcrystalline silicon field-effect transistors (Invited Paper) [6658-12]

S. J. Konezny, École Polytechnique Fédérale de Lausanne (Switzerland); M. N. Bussac, Ctr. de Physique Théorique, CNRS, École Polytechnique (France); A. Geiser, L. Zuppiroli, École Polytechnique Fédérale de Lausanne (Switzerland)

6658 OE Current conduction in ambipolar organic field-effect transistors (OFETs) [6658-13]

H. L. Kwok, Univ. of Victoria (Canada) 


\section{SESSION 4 ORGANIC CRYSTALS}

6658 OG Modification of charge transport in single crystal rubrene (Invited Paper) [6658-15]

O. Mitrofanov, Alcatel-Lucent (USA); D. V. Lang, Columbia Univ. (USA); T. Siegrist, Alcatel-

Lucent (USA); W.-Y. So, Columbia Univ. (USA); C. Kloc, A. P. Ramirez, Alcatel-Lucent (USA)

\section{SESSION $5 \quad$ MOLECULAR DESIGN}

6658 OL Crystal design for organic semiconductors: the effects of substitution on crystal packing (Invited Paper) [6658-20]

J. E. Anthony, B. Purushothaman, Univ. of Kentucky (USA)

6658 ON High performance n-type FETs based on heterocyclic ring systems with trifluoromethylphenyl groups (Invited Paper) [6658-22]

Y. Yamashita, S. Shimono, T. Kono, D. Kumaki, J. Nishida, Tokyo Institute of Technology (Japan); S. Tokito, NHK Science and Technical Research Labs. (Japan)

\section{SESSION 6 OTFTs I}

6658 OP Microstructural mobility of the polymeric gate insulator affecting pentacene charge transport (Invited Paper) [6658-05]

A. Facchetti, C. Kim, T. J. Marks, Northwestern Univ. (USA)

6658 OR Organic phototransistor behavior and light-accelerated bias stress [6658-26]

M. Debucquoy, IMEC (Belgium) and Katholieke Univ. Leuven (Belgium); S. Verlaak, IMEC (Belgium); S. Stoedel, IMEC (Belgium) and Katholieke Univ. Leuven (Belgium); K. Myny, J. Genoe, IMEC (Belgium); P. Heremans, IMEC (Belgium) and Katholieke Univ. Leuven (Belgium)

6658 OS High mobility solution-processed n-channel organic thin film transistors [6658-27] H. Yan, Polyera Corp. (USA); S. LU, Northwestern Univ. (USA); Y. Zheng, P. Inagaki, Polyera Corp. (USA); A. Facchetti, T. J. Marks, Northwestern Univ. (USA)

6658 OT High performance organic field-effect transistors using high-K dielectrics grown by atomic layer deposition (ALD) [6658-28]

X.-H. Zhang, B. Domercq, X. Wang, S. Yoo, T. Kondo, Z. L. Wang, B. Kippelen, Georgia Institute of Technology (USA)

\section{SESSION 7 OTFTs II}

6658 OV Distinguishing between nonlinear channel transport and contact effects in organic FETs [6658-30]

B. H. Hamadani, J. L. LeBoeuf, R. J. Kline, National Institute of Standards and Technology (USA); I. McCulloch, M. Heeney, Merck Chemicals (United Kingdom); C. A. Richter,

L. J. Richter, D. J. Gundlach, National Institute of Standards and Technology (USA) 
6658 OW Dielectric interface modification by UV irradiation: a novel method to control OFET charge carrier transport properties [6658-31]

N. Benson, M. Schidleja, C. Siol, C. Melzer, H. von Seggern, Univ. of Technology Darmstadt (Germany)

6658 OY Transfer printing as a method for fabricating hybrid devices on flexible substrates [6658-33] D. R. Hines, A. E. Southard, A. Tunnell, V. Sangwan, T. Moore, J.-H. Chen, M. S. Fuhrer, E. D. Williams, Univ. of Maryland, College Park (USA)

\section{SESSION 8 NOVEL PROCESSING OF SOLUBLE ORGANIC SEMICONDUCTORS}

665810 Record high mobilities for regioregular poly(3-octylthiophene) (Invited Paper) [6658-35] G. Sauvé, J. Liu, R. Zhang, T. Kowalewski, R. D. McCullough, Carnegie Mellon Univ. (USA)

665811 Ink-jet printing of self-aligned soluble-pentacene crystals for high-performance organic field-effect transistors [6658-36]

J. A. Lim, W. H. Lee, Y. D. Park, H. S. Lee, K. Cho, Pohang Univ. of Science and Technology (South Korea)

665812 Solubility- and temperature-driven thin film structures of polymeric thiophene derivatives for high performance OFET applications [6658-37]

S. W. LeFevre, Rensselaer Polytechnic Institute (USA); Z. Bao, Stanford Univ. (USA); C. Y. Ryu, R. W. Siegel, H. Yang, Rensselaer Polytechnic Institute (USA)

665816 Performance improvement and evaluation of an all plastic organic field effect transistor [6658-41]

S. Ochiai, Aichi Institute of Technology (Japan); X. Wang, Consultant (China); N. P. Rajesh, SSN College of Engineering (India); A. Ohashi, K. Kojima, T. Mizutani, Aichi Institute of Technology (Japan)

\section{POSTER SESSION}

665818 Synthesis of bis-silylated oligothiophenes for solution-processable organic field effect transistors [6658-45]

J. H. Choi, D. W. Cho, S.-H. Jin, Pusan National Univ. (South Korea); C. K. Song, Dong-A Univ. (South Korea); U. C. Yoon, Pusan National Univ. (South Korea)

665819 A novel structure of directly patterned isolating layer for organic thin-film transistor-driven organic light emitting diodes [6658-46]

Y.-K. Wang, T.-H. Lin, J.-Y. Yan, T.-W. Lee, Y.-Y. Shen, S.-T. Yeh, M.-R. Tseng, P.-S. Wu, K.-T. Lin, C.-H. Chen, J.-C. Ho, Industrial Technology Research Institute (Taiwan)

6658 1A Organic field-effect transistors with solution-processible thiophene/phenylene basedoligomer derivative films [6658-47]

H. Yang, Rensselaer Polytechnic Institute (USA); J. Locklin, Univ. of Georgia (USA); B. Singh, Johannes Kepler Univ. of Linz (Austria); Z. Bao, Stanford Univ. (USA)

6658 1B Understanding the mechanisms of photodecarbonylation of the photoprecursors of higher poly(acene)s [6658-48]

R. Mondal, A. N. Okhrimenko, B. K. Shah, D. C. Neckers, Bowling Green State Univ. (USA) 
6658 1C Highly sensitive thin film polymer phototransistors [6658-49]

X. Wang, K. Wasapinyokul, W. D. Tan, R. Rawcliffe, A. J. Campbell, D. D. C. Bradley, Imperial College London (United Kingdom)

$6658 \mathrm{IF}$ Organic thin-film transistors using suspended source/drain electrode structure [6658-53] Y. U. Lee, Y.-H. Kim, J.-I. Han, Korea Electronics Technology Institute (South Korea);

M.-K. Han, Seoul National Univ. (South Korea)

Author Index 


\title{
Conference Committee
}

\author{
Symposium Chair \\ Zakya H. Kafafi, Naval Research Laboratory (USA) \\ Conference Chairs \\ Zhenan Bao, Stanford University (USA) \\ David J. Gundlach, National Institute of Standards and Technology \\ (USA) \\ Program Committee \\ Kilwon Cho, Pohang University of Science and Technology (South \\ Korea) \\ Dean M. DeLongchamp, National Institute of Standards and \\ Technology (USA) \\ C. Daniel Frisbie, University of Minnesota (USA) \\ Thomas N. Jackson, The Pennsylvania State University (USA) \\ Hagen Klauk, Max-Planck-Institut für Festkörperforschung (Germany) \\ Sang Yoon Lee, Samsung Advanced Institute of Technology (South \\ Korea) \\ Iain A. McCulloch, Merck Chemicals Ltd. (United Kingdom) \\ Alberto Salleo, Stanford University (USA) \\ Takao Someya, The University of Tokyo (Japan)
}

\section{Session Chairs}

1 Circuits and Displays

Thokchom B. Singh, Johannes Kepler Universität Linz (Austria)

2 Gate Dielectrics and Contacts

John E. Anthony, University of Kentucky (USA)

3 Charge Transport

David J. Gundlach, National Institute of Standards and Technology (USA)

4 Organic Crystals

Daniel A. Moses, University of California, Santa Barbara (USA)

5 Molecular Design

Antonio F. Facchetti, Northwestern University (USA) 
OTFTs I

Gerwin H. Gelinck, TNO (Netherlands)

7 OTFTs II

Kilwon Cho, Pohang University of Science and Technology (South Korea)

$8 \quad$ Novel Processing of Soluble Organic Semiconductors

Gerwin H. Gelinck, TNO (Netherlands) 